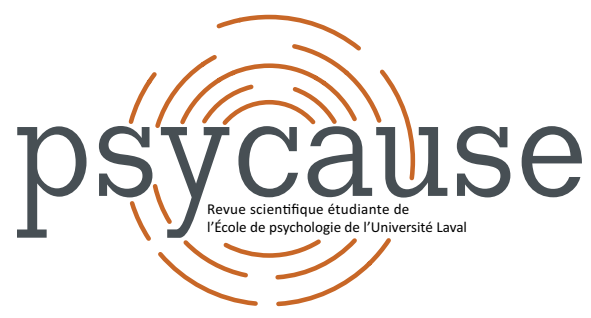

\title{
PSYCAUSE
}

Revue scientifique étudiante de l'École de psychologie de l'Université Laval

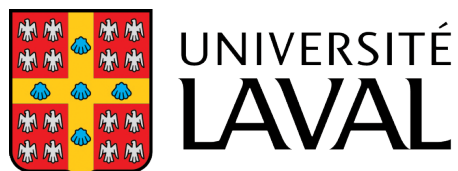

Faculté des sciences sociales École de psychologie

\section{LANGUE D'ORIGINE ET EXPÉRIENCE IDENTITAIRE CHEZ LES CANADIENS DE DEUXIĖME GÉNÉRATION}

Milica MILJUS ${ }^{1, \star}$, Frédérique MONTREUIL ${ }^{1}$, Laurence GARCEAU ${ }^{1}$, Jennifer TAING ${ }^{1}$ et Maya A. YAMPOLSKY'

${ }^{1}$ École de psychologie, Université Laval

*milica.miljus.1@ulaval.ca

\section{Pour citer l'article}

Miljus, M., Montreuil, F., Garceau, L., Taing, J., \& Yampolsky, M. A. (2019). Langue d'origine et expérience identitaire chez les Canadiens de deuxième génération. Psycause: Revue scientifique étudiante de l'École de psychologie de l'Université Laval, 9(2), 17-19. 
d'occurrence des pensées négatives explique une portion de la relation positive entre le recours à l'évitement expérientiel ainsi qu'à la suppression émotionnelle, et la PRC. Les résultats suggèrent aussi que la fréquence d'occurrence des pensées positives explique une partie de la relation positive entre le recours à la suppression émotionnelle et la PRC. Ces observations sont cohérentes avec celles de David, Montgomery et Bovbjerg (2006) qui montrent que le pessimisme et l'optimisme dispositionnel médient la relation entre les stratégies de RE et la détresse psychologique chez ces patientes atteintes. Ainsi, il semble que les stratégies de RE utilisées par les participantes pour agir sur leur PRC les amèneraient à modifier le type et la fréquence d'occurrence de leurs pensées.

En somme, ces résultats remettent en doute les effets bénéfiques de la pensée positive dans l'adaptation psychologique au cancer et identifient un mécanisme possible par lequel les stratégies de RE et les pensées accroîtraient le niveau de sévérité de la PRC chez les femmes atteintes d'un cancer du sein. Ils appuient la pertinence de s'intéresser à l'efficacité des interventions cognitives-comportementales pour diminuer la PRC en soulignant l'importance des interprétations cognitives. D'autres études seront nécessaires afin de mieux comprendre les relations entre ces variables.

\section{Références}

David, D., Montgomery, G. H., \& Bovbjerg, D. H. (2006). Relations between coping responses and optimism-pessimism in predicting anticipatory psychological distress in surgical breast cancer patients. Personality and Individual Differences, 40(2), 203-213. doi:10.1016/j.paid.2005.05.018

Deimling, G. T., Bowman, K. F., Sterns, S., Wagner, L. J., \& Kahana, B. (2006). Cancer-related health worries and psychological distress among older adult, long-term cancer survivors. Psycho-Oncology, 15(4), 306-320. doi:10.1002/ pon.955

Gross, J. J., \& Thompson, R. A. (2007). Emotion Regulation: Conceptual Foundations. New York, NY: Guilford Press.

Hurt, C. S., Weinman, J., Lee, R., \& Brown, R. G. (2012). The relationship of depression and disease stage to patient perceptions of Parkinson's disease. Journal of Health Psychology, 17(7), 1076-1088. doi: 10.1177/1359105311428537

Lebel, S., Ozakinci, G., Humphris, G., Mutsaers, B., Thewes, B., Prins, J., Dinkel, A., \& Butow, P. (2016). From normal response to clinical problem: definition and clinical features of fear of cancer recurrence. Supportive Care in Cancer, 24(8), 3265-3268. doi:10.1007/s00520-016-3272-5

Savard, J. (2010). Faire face au cancer avec la pensée réaliste. Montréal, QC: Flammarion.

\title{
Pour citer l'article
}

Dallaire-Habel, S., Ouellet, G., Thériault-Couture, F., Massicotte, V., Ivers, H., Guimond, A.-J., \& Savard, J. (2019). La relation entre les pensées positives et négatives, les stratégies de régulation émotionnelle et la peur de récidive du cancer. Psycause: Revue scientifique étudiante de l'École de psychologie de l'Université Laval, 9(2), 15-17.

\section{LANGUE D'ORIGINE ET EXPÉRIENCE IDENTITAIRE CHEZ LES CANADIENS DE DEUXIÈME GÉNÉRATION}

\author{
Milica MILJUS ${ }^{1}$ $*$, Frédérique MONTREUIL ${ }^{1}$, Laurence GARCEAU ${ }^{1}$, Jennifer TAING ${ }^{1}$ et Maya A. YAMPOLSKY ${ }^{1}$ \\ ${ }^{1}$ École de psychologie, Université Laval \\ *milica.miljus.1@ulaval.ca
}

\section{Mots clés : Langue d'origine; Deuxième génération; Identité; Sentiment d'appartenance}

Les individus de deuxième génération sont définis comme étant nés au pays et ayant au moins un parent migrant (Nesteruk, Helmstetter, Gramescu, Siyam \& Price, 2015). Les enfants qui ont immigré avant l'âge de cinq ans sont inclus dans cette définition, ayant été socialisés dans la culture majoritaire de façon similaire à ceux nés au pays (Nesteruk et coll., 2015). Les individus de deuxième génération tendent à adopter la langue majoritaire par le biais de l'environnement social (p. ex., à l'école), et ce, au détriment du maintien ou de l'apprentissage de la langue d'origine (Fillmore, 1991). Sachant que la connaissance de la langue d'origine joue un rôle dans le développement et le renforcement de l'identité culturelle (Phinney, Romero, Nava \& Huang, 2001), cette recherche qualitative vise à explorer les relations entre l'apprentissage de la langue, le sentiment d'appartenance et l'expérience identitaire des Canadiens de deuxième génération et à explorer les variables contextuelles associées. 


\section{Méthode}

Les participants ont été recrutés via les médias sociaux, le bouche-à-oreille et Prolific Academic. L'échantillon final comprend 50 adultes canadiens issus de la deuxième génération. Ils ont rempli un questionnaire sociodémographique et une question ouverte: "Veuillez nous en dire davantage sur vos expériences concernant: Votre apprentissage et utilisation de votre/vos langue(s) d'origine; Comment cela est associé à votre sentiment d'appartenance et à vos identités culturelles.»

Afin de dégager la signification des extraits, une analyse de contenu a été effectuée. Trois juges indépendants, les auteurs de cette étude, ont effectué le codage à la suite de formations pour assurer l'accord interjuges selon le $\mathrm{Pi}$ de Scott $(\pi=.84)$. Le programme QDA Miner 5.0.21 de Recherches Provalis (2011) a permis de dégager les principaux concepts exprimés par l'analyse de fréquence de codage et d'examiner la concomitance entre des concepts à l'intérieur d'une même réponse à l'aide de l'analyse de cooccurrence.

\section{Résultats et discussion}

Les modes d'apprentissage de la langue d'origine les plus fréquents dans l'échantillon sont l'école de langue et les parents. Une réticence face à l'apprentissage est parfois associée à l'école de langues par les participants, mais celle-ci est aussi fréquemment combinée à un apprentissage efficace. La participation des individus de deuxième génération au sein de ces écoles est généralement forcée par les parents et survient habituellement la fin de semaine selon les participants interrogés, ce qui pourrait expliquer cette réticence. Toutefois, certains participants rapportent être reconnaissants à l'âge adulte d'avoir appris la langue d'origine, ce qui suppose que la réticence n'empêcherait ni l'acquisition de la connaissance de la langue d'origine, ni son appréciation à l'âge adulte. L'apprentissage, même forcé, paraît donc être positif à long terme chez les participants.

Certains participants mentionnent un apprentissage efficace de la langue d'origine en contiguïté avec un sentiment d'appartenance à la culture d'origine, tel que le décrit le participant 12:

Je crois fermement que parler espagnol a eu une influence sur mon sentiment d'appartenance [...]. Cela m'a permis de connecter aux autres membres de mon groupe culturel [...] et d'expérimenter la culture.

En contraste, un sentiment de faible appartenance semble avoir été davantage rapporté par les participants relatant un apprentissage inefficace de la langue d'origine. Le participant 23 exprime cette relation:
Nous avions des classes d'Inuktitut, mais ce n'est jamais resté [...]. Je sens que parce que je n'ai jamais pu m'immerger dans la langue, [...] j'ai une connexion plus faible avec mon héritage que les autres personnes de mon âge.

La connaissance ou non de la langue d'origine affecterait donc l'accès à certains objets représentatifs de la culture d'origine, comme des interactions avec les autres membres du groupe, ce qui en retour influencerait la force du sentiment d'appartenance à cette culture.

Le manque de connaissance de la langue d'origine entrave également l'identité culturelle chez quelques participants. Cela se reflète par une cooccurrence entre l'apprentissage inefficace de la langue d'origine et l'intériorisation du déni des autres envers son identité culturelle d'origine. Le déni de l'identité correspond à la perception d'autrui qu'un individu ne répond pas à certains critères stéréotypés nécessaires afin de le considérer comme membre d'une culture donnée (Cheryan \& Monin, 2005). Un de ces critères semble être la connaissance de la langue, car certains participants intériorisent le déni d'autrui et ne croient pas être un réel membre de leur culture d'origine lorsqu'ils considèrent leur compétence langagière comme insuffisante. Certains rapportent également de l'embarras et de la gêne face au manque de connaissance langagière, ce qui illustre un tourment dans le fait de ne pas connaître la langue, tel que présenté par le participant 37 :

\section{Je me sens embarrassé quand je ne peux pas com- mander de nourriture aux restaurants, comme si j'étais une personne chinoise whitewashed, et je me sens un peu comme une fraude dans ces situations.}

En bref, l'apprentissage efficace de la langue d'origine joue un rôle bénéfique dans l'accès à la culture d'origine, dans le sentiment d'appartenance et dans l'identité culturelle d'origine. Les bénéfices observés sont cohérents avec ceux mentionnés dans les études antérieures (p. ex., sur le sentiment d'appartenance, Nesteruk et coll., 2015). Un élément nouveau amené par l'étude est l'importance des écoles de langues dans l'acquisition de la langue, qui s'est avéré être un mode d'apprentissage plus mentionné que les parents.

\section{Références}

Cheryan, S., \& Monin, B. (2005). Where are you really from?: Asian Americans and identity denial. Journal of Personality and Social Psychology, 89(5), 717 730. doi:10.1037/00223514.89.5.717

Fillmore, L. W. (1991). When learning a second language means losing the first. Early Childhood Research Quarterly, 6(3), 323 346. doi:10.1016/S0885-2006(05)80059-6 
Nesteruk, O., Helmstetter, N.-M., Gramescu, A., Siyam, M. H., \& Price, C. A. (2015). Development of ethnic identity in young adults from immigrant families: "I want to hold onto my roots, but I also want to experience new routes". Marriage \& Family Review, 51(5), 466 487. doi:10.1080/01494 929.2015.1060288
Phinney, J. S., Romero, I., Nava, M., \& Huang, D. (2001). The role of language, parents, and peers in ethnic identity among adolescents in immigrant families. Journal of Youth and Adolescence, 30(2), 135 153. doi:10.1023/A:1010389607319

\section{Pour citer l'article}

Miljus, M., Montreuil, F., Garceau, L., Taing, J., \& Yampolsky, M. A. (2019). Langue d'origine et expérience identitaire chez les Canadiens de deuxième génération. Psycause: Revue scientifique étudiante de l'École de psychologie de l'Université Laval, 9(2), 17-19.

\section{LIEN ENTRE LE STRESS PARENTAL ET LA SENSIBILITÉ MATERNELLE ENTRE SIX ET 18 MOIS}

Catherine POVEDA PERDOMO ${ }^{1,4, *}$, Claudia BERGERON ${ }^{1,4}$, Mathilde YERGEAU ${ }^{1,4}$, Laura-Émilie SAVAGE ${ }^{1,4}$, Audrey GAUTHIER-LÉGARÉ ${ }^{1,4}$, Jean-Pascal LEMELIN ${ }^{3,4}$, Marc PROVOST ${ }^{2}$ et George M. TARABULSY1,4

${ }^{1}$ École de psychologie, Université Laval; ${ }^{2}$ Département de psychologie, Université du Québec à Trois-Rivières; ${ }^{3}$ Faculté d'éducation, Université de Sherbrooke; ${ }^{4}$ Centre de recherche universitaire sur les jeunes et les familles (CRUJeF)

* catherine.poveda-perdomo.1@ulaval.ca

\section{Mots-clés : Sensibilité maternelle; Stress parental; Développement de l'enfant}

La sensibilité maternelle (SM) réfère à l'habileté de la mère à percevoir et à interpréter les signaux de son enfant, ses comportements et ses émotions, ainsi que d'y répondre rapidement et de façon appropriée (Ainsworth, 1982; Neuhauser, 2018). La SM est un des plus importants médiateurs postnatals du développement, puisqu'elle est associée à différentes sphères du développement de l'enfant (Tarabulsy, Moran, Pederson, Provost \& Larose, 2011). Plusieurs études identifient le stress parental (SP) comme étant un important corrélat de la SM. Le SP se définit comme l'écart ressenti par le parent, d'une part entre les exigences posées par la situation de la parentalité et par l'enfant lui-même, et d'autre part, par les ressources et les habiletés disponibles pour répondre à ces exigences (Lacharité, Ethier \& Couture, 1996). La plupart des études démontrent que le SP et la SM sont inversement corrélés (c.-à-d. que le fait de vivre plus de SP est lié à une baisse de la SM; Neuhauser, 2018). Il est possible qu'une SM moindre engendre des situations de stress pour les parents. Or, la plupart des travaux utilisent des devis transversaux et ne permettent donc pas de connaître la direction du lien (Booth \& coll., 2018). Cette étude a pour premier objectif d'examiner les liens entre le $\mathrm{SP}$ et la SM lors d'interactions mère-enfant à six, 10, 15 et 18 mois. Le deuxième objectif est de vérifier les liens longitudinaux entre les concepts, en tenant compte de la contribution des variables au temps précédent.

\section{Méthode}

Les données pour les quatre temps de mesures sont recueillies auprès de 83 dyades et les analyses sont effectuées sur les 160 dyades recrutées avec la méthode de traitement des données manquantes du maximum de vraisemblance. L'échantillon d'enfants comprend 89 filles et 71 garçons. Le SP est évalué à l'aide du Parenting Stress Index (PSI - short form, Abidin, 1995) qui mesure la détresse parentale, les interactions dysfonctionnelles et les difficultés du nourrisson. La SM est évaluée à l'aide du Maternal Behavior Q-set (MBOS; Pederson \& Moran, 1995), une mesure observationnelle de ce construit. Des rencontres à la maison ont été réalisées lorsque les enfants étaient âgés de six, 10,15 et 18 mois pour compléter ces deux mesures.

\section{Résultats et discussion}

Concernant le premier objectif, des corrélations de Pearson montrent que contrairement à ce que la littérature rapporte, le SP et la SM ont une très grande stabilité et que leur évolution dans le temps semble indépendante l'une de l'autre, particulièrement à six et 10 mois, où les concepts ne sont pas liés entre eux. Des corrélations modérées apparaissent à 15 et 18 mois et pourraient être expliquées par des changements dans les demandes de l'enfant, dans son développement ou dans l'écologie développementale durant sa deuxième année de vie. Cependant, le fait saillant de 